\title{
Single Burst Depth-Resolving Raman Spectrometer Based on a SPAD Array with an On-Chip TDC to Analyse Heterogenous Liquid Samples
}

\author{
Jere Kekkonen \\ Circuits and Systems Research Unit \\ University of Oulu \\ Oulu, Finland \\ jere.kekkonen@oulu.fi
}

\author{
Ilkka Nissinen \\ Circuits and Systems Research Unit \\ University of Oulu \\ Oulu, Finland \\ ilkka.nissinen@oulu.fi
}

\begin{abstract}
A single burst depth-resolving Raman spectrometer based on a CMOS single-photon avalanche diode (SPAD) array with an on-chip 256-channel time-to-digital converter (TDC) has been developed to analyze heterogenous liquid sample without the need for any additional off-chip delay circuits. The depth derivation of Raman sensitive layers on top of and under a water phase is based on measuring the time-ofarrivals of Raman scattered photons generated with short laser pulses ( $\sim 150 \mathrm{ps})$ by utilizing the 256 TDCs at every 256 spectral points. Raman spectra of different layers is derived by postprocessing the time domain histograms with the resolution of 200 ps corresponding to $23 \mathrm{~mm}$ depth in water. The minimum depth difference between two layers that could be independently identified was $65 \mathrm{~mm}$ this being limited by the full-width value of the instrument response function of the whole system $(570 \mathrm{ps})$. The depth derivation error was measured to be less than $19 \mathrm{~mm}$. This technology could enable a compact and fast depthresolving Raman spectrometer to be used in potential applications within the fields of food, pharmaceuticals and beverage industries, for example.
\end{abstract}

Keywords-SPAD detector, on-chip time-to-digital converter, Raman spectroscopy, time-correlated single-photon counting, Raman depth-profiling

\section{INTRODUCTION}

Raman spectroscopy is a widely used optical spectroscopy technique to solve the chemical structure of different kind of samples regardless of their state of matter. Raman scattered photons are shifted from the excitation wavelength when samples are illuminated by a monochromatic laser source and these shifts are unique for each chemical component $[1,2]$. The intensity distribution as a function of wavelength or wavenumber can be recorded by a line sensor under a spectrograph or a grating resulting in a chemical fingerprint of the measured sample. In addition, Raman spectroscopy is a practical method to study aqueous samples because it has low sensitivity for water [3].

Pulsed laser sources instead of traditional continuous wave lasers can be utilized with gated-detectors to suppress effectively the main rival of Raman scattering, fluorescence $[4,5,6]$. The fluorescence suppression is based on the different time responses of Raman scattered photons and fluorescence photons. Raman photons are scattered during the laser pulse (normal pulse width $100 \mathrm{ps}-1 \mathrm{~ns}$ ) but the fluorescence photons have exponentially decaying probability density function with the time constants from nanoseconds to even microseconds depending on the sample. The fluorescence background can be effectively suppressed if a detector is enabled to collect the photons only during a short laser pulse $[5,6]$.

This work was supported by the Academy of Finland (Centre of Excellence in Laser Scanning Research) under Contract 307362 and Contracts 314404 and 292609.
Pulsed laser sources with gated detectors can be also utilized to solve molecular depth structure of multilayer samples based on the fact that the flight time of Raman scattered photons from a deeper layer is longer than from a top layer as illustrated in Fig. 1 (a) and (b), where the block diagram of a Raman depth-profiling spectrometer and its timing diagram at a single spectral point are shown, respectively [7]. For example, when the Raman scattered photons from the top layer are wanted to be collected by the gated detector, the delay between an emitted laser pulse, which also generates the trigger signal, and the start time of the time gate is adjusted by means of the delay unit to be $T_{1}$ enabling the gated detector to collect only the Raman scattered photons arriving from the top layer (red colored pulse) as shown in Fig. 1. The delay value of $\mathrm{T}_{2}$ is used when the Raman scattered photons from the bottom layer are wanted to be collected. To identify independently the Raman spectra of thin adjacent layers, the widths of the time gate and a laser pulse must be short enough $(\sim 100 \mathrm{ps})$ [8]. Otherwise, the Raman spectra of adjacent layers will be merged together as illustrated in Fig. 1 (b) where the time gate with the delay value of $T_{1}$ is collecting also some of the Raman photons from the bottom layer. It must be underlined that in Fig. 1 (b) is illustrated only the timing diagram at a single spectral point and in a line sensor, every spectral point will collect the time domain distributions.

The main aim of present depth-profiling technologies has been to identify the molecular depth structure of two-layer samples nor to solve the actual depth of different layers. These devices have been based on a Kerr gate with a detector and time-gated CCD [7,9], but the complexity of these devices has hindered their widespread usage. Two methods, the use of a time-correlated single-photon counting (TCSPC) silicon photomultiplier detector and a TCSPC camera, to reduce the complexity were presented in $[10,11]$. The former one requires additional acquisition electronics because of the limited integration capability of a microchannel plate-based photomultiplier tubes and the latter one has a single-pixel detector leading to long measurement time when the wide spectral range is needed to be measured.

CMOS technology-based single-photon avalanche diodes (SPAD) has been also used to measure Raman spectra and effectively suppress fluorescence background with a pulsed laser $[12,13]$. In addition, CMOS technologies have made it possible to fabricate SPAD arrays on the same chip together with gating electronics to increase the integration level of time-correlated Raman spectrometers $[14,15,16]$. In addition, the depth-resolving Raman spectrometer has been developed by using a time-correlated $16 \times 256$ CMOS SPAD line sensor and an accurate off-chip delay unit to sweep the time gate 


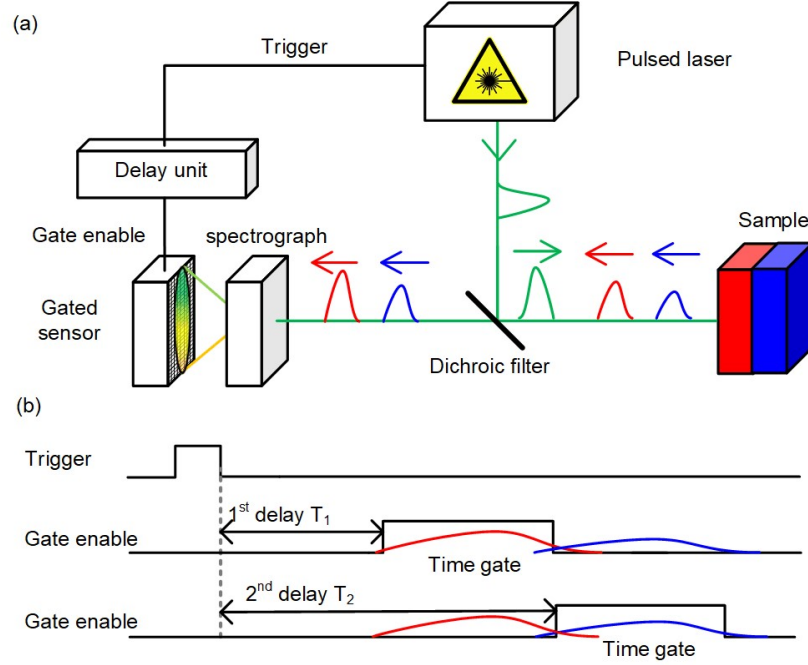

Fig. 1. The (a) block digram of a Raman depth-profiling spectrometer and (b) its timing digram at a single spectral point.

position of that sensor [8]. The variability of depth derivation was measured to be $\pm 0.43 \mathrm{~cm}$ with that setup when semitransparent media were probed. Even though that setup was capable to derive the depths of different molecules inside a semitransparent media, the technique was time-consuming because it needed a new burst of laser pulses at every time gate position, i.e. at every depth.

This paper presents a single burst depth-resolving Raman spectrometer based on the same $16 \times 256$ SPAD array with an on-chip 256-channel time-to-digital (TDC) converter that was used as the line sensor in $[8,16]$ but here the resolution of the on-chip TDC has been expanded in order to resolve the Raman depth profiles of around ten centimeter deep heterogeneous liquid samples with actual depth information by using only a single burst of laser pulses. This enables the depth profiling of those samples rapidly without any additional off-chip delay unit and thus, the integration level and the measurement speed of the whole device can be increased dramatically. The time resolution and time range of the sensor were set to $200 \mathrm{ps}$ and $1200 \mathrm{ps}$, respectively, and the depth derivation is based on the post-processing of the time domain data of backscattered Raman photons simultaneously recorded from the whole centimeter-scale liquid sample.

The main aim of this work is to show the functionality of a fully integrated TCSPC CMOS sensor in depth-resolving Raman spectroscopy without the need for any additional offchip delay units. This technology could make it possible to derive rapidly the Raman depth profiles of liquids and other semitransparent media with actual depth information by using only a single burst of laser pulses. This kind of fast and compact depth-resolving Raman spectrometer could open new application possibilities, for example, in quality control in the food and beverage industry and the studying of natural and mining waters.

\section{The SINGLE BURST DePTH-RESOlVING RAMAN SPECTROMETER AND TEST METHODS}

\section{A. Single burst depth-resolving Raman spectrometer based} on a 16×256 SPAD array with a 256-channel TDC

The block and principled timing diagram of the single burst depth-resolving Raman spectrometer based on a $16 \times 256$ SPAD array with an on-chip 256-channel TDC are shown in

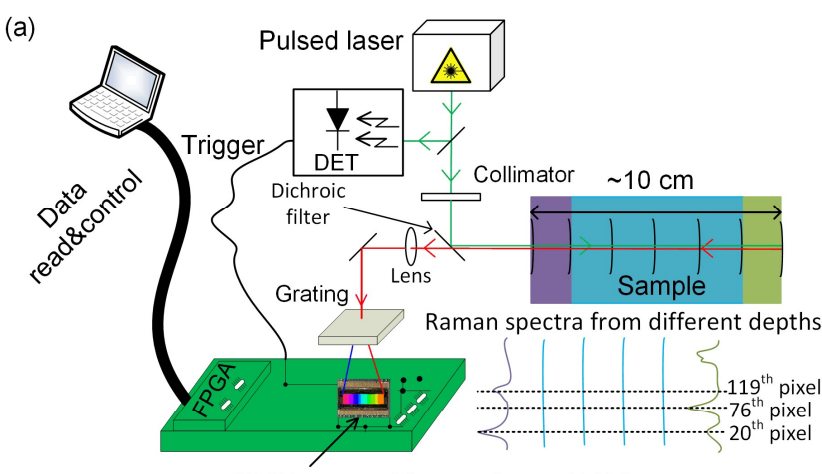

16x256 SPAD array with 256-channel TDC

(b)

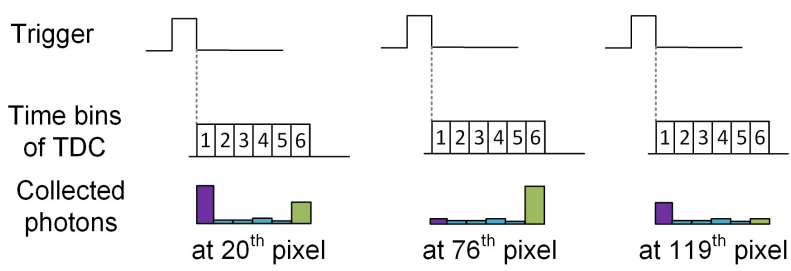

Fig. 2. The (a) block and (b) timing diagrams of the single burst depthresolving Raman spectrometer at the three different pixels, i.e. wavenumbers.

Fig. 2 (a) and (b), respectively. The laser used is a $532 \mathrm{~nm}$ pulsed laser (Teem Photonics, model ANG500P-CHS) having the pulse width, pulse energy, pulse rate and bandwidth of 150 ps (FWHM), $0.6 \mu \mathrm{J}, 350 \mathrm{kHz}$ and $0.11 \mathrm{~nm}$, respectively. The burst of laser pulses is shot to the heterogeneous liquid sample that has, for example, two Raman sensitive layers at the top and at the bottom of the sample (purple and green layers in Fig. 2 (a)). A small part of the laser pulses are delivered to an optical detector (DET in Fig. 2 (a)) to generate a trigger signal for the SPAD line sensor in order to bias all the SPADs to Geiger mode and to start the time-of-arrival (ToA) measurement of the photons scattered from the sample across the whole spectral range at once ( 256 spectral point i.e. pixels in spectral axis). Because the photon detection probability is markedly less than one in Raman spectroscopy, the measurement principle here is similar as in any other TCSPC technique. During the burst of laser pulses (several hundreds of thousands of laser pulses) the ToA of photons are recorded within the six time bins of the 256 TDCs having the resolution of 200 ps (note that Raleigh scattered photons are filtered by the dichroic filter before grating). For example, the time domain distribution of the collected photons at the $20^{\text {th }}$ pixel shows that the photon counts of the bins 1 and 6 are larger than the photon counts of the bins $2-5$ because both of the Raman sensitive layers have Raman peak at the $20^{\text {th }}$ pixel as shown in Fig. 2 (a) (Raman spectra from different depths). Bins 2-5 are just collecting the background from the non-Raman sensitive layer. Fig. 2 (b) shows also the time domain distributions of collected photons at the $76^{\text {th }}$ and $119^{\text {th }}$ pixels to clarify the simultaneous operation across the whole spectral range. In total 256 time domain distribution are recorded simultaneously by the 256 TDCs within the time range of 1200 ps and thus, the Raman spectra at the different depths can be derived by plotting the photon count of each time bin as a function of the pixels. A field-programmable gate array (FBGA in Fig. 2(a)) is used for control and data read-out.

The $16 \times 256$ SPAD array with the on-chip 256-channel TDC was fabricated in a $0.35 \mu \mathrm{m}$ high voltage CMOS technology [16]. The simplified block and timing diagrams of 


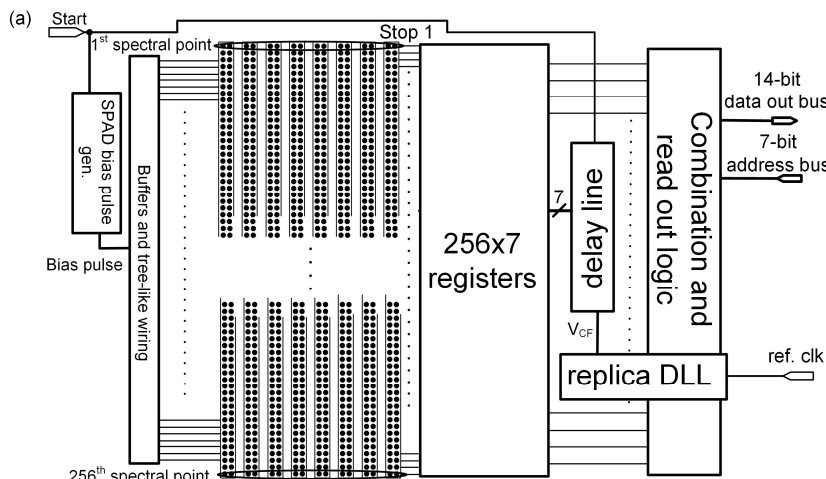

(b)

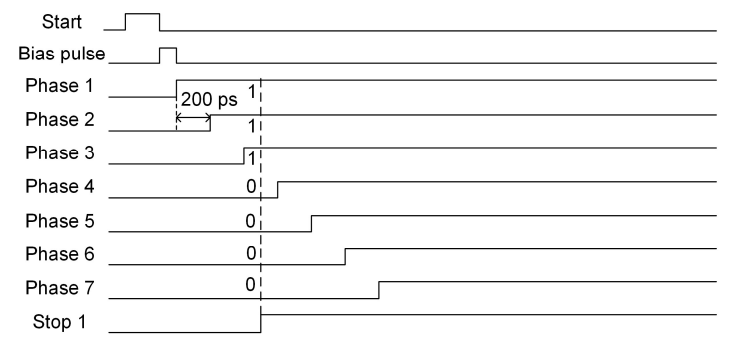

Fig. 3. The (a) block and (b) timing diagrams of the $16 \times 256$ SPAD array with the on-chip 256-channel TDC.

the SPAD array with the on-chip TDC are shown in Fig. 3 (a) and (b), respectively. The trigger signal from the laser (start in Figs. 3) is used to generate a logic level bias pulse for all the SPADs simultaneously in order to charge the voltages of the SPADs over their breakdown voltage (the excess bias of SPADs is approximately $3.3 \mathrm{~V}$ ). The same trigger signal is delayed by a delay line generating seven phases (Phase 1Phase 7 in Fig. 3 (b)) with the delay difference of 200 ps between adjacent phases. This delay value is locked by a replica delay-locked loop (replica DLL in Fig. 3 (a)) to offchip oscillator having the clock frequency of $25 \mathrm{MHz}$. The states of the phases of the delay line at every spectral point are stored to $256 \times 7$ registers by the detected photons. For example, Fig. 3 (b) shows the situation at the first spectral point where a photon arriving between phase 3 and phase 4 (Stop 1) is storing the state of 0000111 to the register corresponding to the ToA of $500 \mathrm{ps}$ when the time-to-digital conversion is centered in the middle of the $3^{\text {rd }}$ time bin. The detailed operation of the SPAD array can be found in [16].

\section{B. Test methods}

In this proof-of-concept study, two different heterogeneous liquid samples were investigated. The first sample consisted of $2.5 \mathrm{~cm}$ layer of sunflower oil on the top of a $7.5 \mathrm{~cm}$ deep water phase as shown in Fig. 4 (a). At the bottom of the sample was a $2.0 \mathrm{~cm}$ high sediment of titanium dioxide $\left(\mathrm{TiO}_{2}\right)$ powder. The second sample also consisted of sunflower and water phases, which height were now $1.0 \mathrm{~cm}$ and $6 \mathrm{~cm}$, respectively, as shown in Figs. 4 (b) and 4 (c) (Fig. 4 (c) shows a photograph from the measurements). At the bottom of the second sample was placed a sintered block of $\mathrm{Ba}\left(\mathrm{Mg}_{1 / 3} \mathrm{Ta}_{2 / 3}\right) \mathrm{O}_{3}$ powder. Reference Raman spectra for sunflower oil, $\mathrm{TiO}_{2}$ and $\mathrm{Ba}\left(\mathrm{Mg}_{1 / 3} \mathrm{Ta}_{2 / 3}\right) \mathrm{O}_{3}$ can be found from $[17,18,19]$, respectively. The laser beam was tilted to vertical by using a mirror and a single laser burst containing 3 million laser pulses were shot to the samples in these measurements resulting in acquisition times of 8.6 seconds with the laser repetition rate of $350 \mathrm{kHz}$.

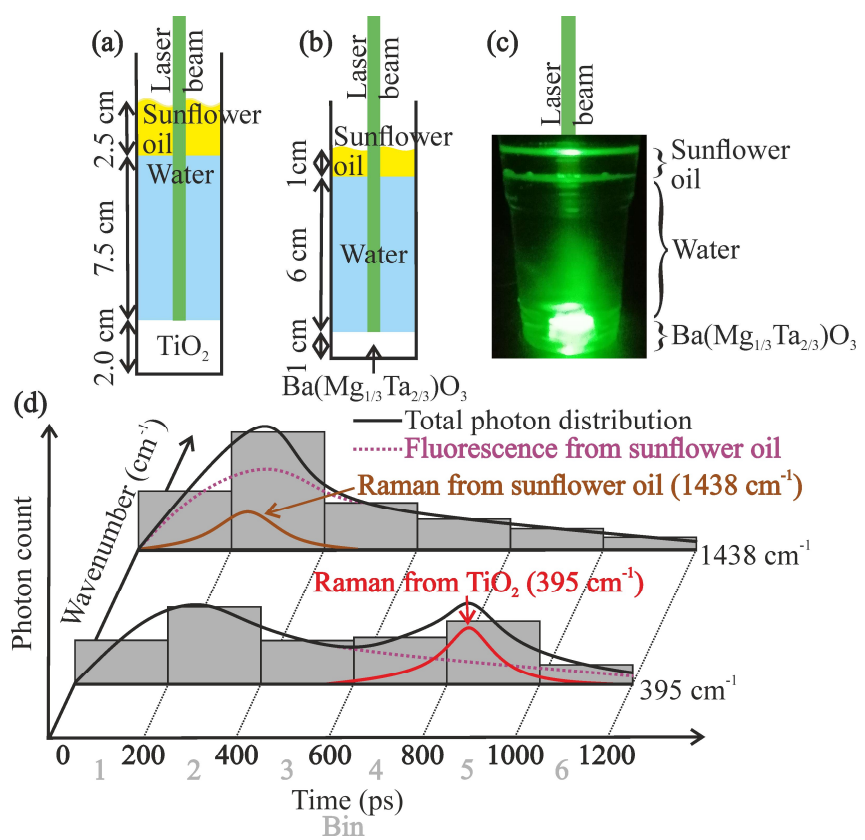

Fig. 4. The (a) first and (b) second sample configurations. (c) Photograph from the measurement of the second sample configuration. (d) Principled Raman, fluorescence, and total photon distributions of the first sample configuration alongside with the principled photon count histograms at two example Raman active spectral points (26 and 185 corresponding to wavenumber values of $1438 \mathrm{~cm}^{-1}$ and $395 \mathrm{~cm}^{-1}$, respectively).

Principled Raman, fluorescence and total photon distributions of the first sample configuration (sunflower oilwater- $\mathrm{TiO}_{2}$ ) are shown in Fig. 4 (d) alongside with the principled photon count histograms at two example Raman active spectral points to depict the basic concept of Raman depth-profiling and depth derivation with the used sensor. The example spectral points were 26 and 185 , which correspond to wavenumber values of $395 \mathrm{~cm}^{-1}$ (strong Raman activity of $\mathrm{TiO}_{2}$ ) and $1438 \mathrm{~cm}^{-1}$ (strong Raman activity of sunflower oil), respectively. As can be seen from Fig. 4 (d), the backscattered Raman photons from the sunflower oil phase (brown line) at the top of the sample reaches the detector earlier than the Raman photons from the $\mathrm{TiO}_{2}$ sediment (red line) at the bottom of the sample simply because of the longer ToA of the Raman photons from $\mathrm{TiO}_{2}$ due to the longer distance. Here the maximum Raman intensity from sunflower oil is observed at the second time bin of the TDC and from $\mathrm{TiO}_{2}$ at the fifth time bin resulting in $600 \mathrm{ps}$ ToA difference between these two layers. This ToA difference can be converted to depth difference with the help of the equation $\Delta \mathrm{d}=\mathrm{v}^{*} \Delta \mathrm{t} / 2$ where the $\mathrm{v}$ is the speed of light in water $\left(\mathrm{v}=2.30^{*} 10^{8} \mathrm{~m} / \mathrm{s}\right.$, computed with $\mathrm{n}=1.3$ ) and $\Delta \mathrm{t}$ is the measured ToA difference. Now the ToA difference of $600 \mathrm{ps}$ converts to the depth difference of $69 \mathrm{~mm}$, which is a little less than the actual depth difference ( $87.5 \mathrm{~mm}$, measured from the middle of the sunflower oil to the surface of $\mathrm{TiO}_{2}$ ) as can be seen from Fig. 4 (a) and this inaccuracy is mostly explained by the 200 ps resolution of the time bins (the accuracy of the method is discussed in more detail at the next section).

From Fig. 4 (d) can be also observed how the high broadband fluorescence of sunflower oil does not only cause challenges to measure the Raman spectrum of sunflower oil itself but the fluorescence tail masks also to some extent the Raman signal from the $\mathrm{TiO}_{2}$ sediment. Nevertheless, the basic concept of fluorescence suppression of time gating technique 
works also for the fluorescence tail. Most of the Raman signal from $\mathrm{TiO}_{2}$ fit inside the fifth bin whereas the fluorescence from sunflower oil covers the whole time range of the TDC. Therefore, by using only the fifth time bin, most of the Raman photons from $\mathrm{TiO}_{2}$ can be collected and at the same time, most of the fluorescence from sunflower oil can be rejected.

\section{MEASUREMENT RESUltS}

Fig. 5 (a), (b), (c) and (d) show the Raman spectra of the first sample, which consisted of water between sunflower oil and $\mathrm{TiO}_{2}$ as showed in Fig. 4 (a), as a function of the time bins of the TDC with the time gate widths of $200 \mathrm{ps}, 400 \mathrm{ps}, 600$ ps and $1200 \mathrm{ps}$, respectively. The resolution of the TDC i.e. the width of a single time bin was set to $200 \mathrm{ps}$ (corresponding to $23 \mathrm{~mm}$ depth in water) and the wider time gate widths were achieved by summing the photon counts of adjacent time bins. As can be seen from Fig. 5 (a), the clearest Raman spectrum of sunflower oil (Raman peaks from $1000 \mathrm{~cm}^{-1}$ to $1800 \mathrm{~cm}^{-1}$ ) is derived by using the second time bin of the TDC. However, the highest Raman peaks of sunflower oil can be already observed within the first time bin due to the width of the instrument response function (IRF) of the whole device, which has been measured to be $225 \mathrm{ps}$ (FWHM) with the laser pulse width of 150 ps (FWHM) resulting in a full-width of $\sim 570$ ps [16]. The Raman spectra of $\mathrm{TiO}_{2}$ derived by using the fourth and fifth time bins are similar indicating that the position of a $\mathrm{TiO}_{2}$ sample locates in the middle of these two time bins. The depth difference between these two layers can be solved straightforwardly by using the method explained in the previous section. The ToA difference between the observed maximum intensities of the layers is 600 ps (900 ps (bin 5)-300 ps (bin 2)) corresponding to the depth difference of $69 \mathrm{~mm}$. The actual depth difference between the layers was measured to be here $87.5 \mathrm{~mm}$. From Figs. 5 (b), (c) and (d) can be observed that the depth difference between these two samples can be also derived with the time gate widths of 400 ps and 600 ps, but the Raman spectra of sunflower oil and $\mathrm{TiO}_{2}$ merge together when the time gate width of $1200 \mathrm{ps}$ is used.

Fig. 6 (a), (b), (c) and (d) show the Raman spectra of the second sample, which consisted of water between sunflower oil and $\mathrm{Ba}\left(\mathrm{Mg}_{1 / 3} \mathrm{Ta}_{2 / 3}\right) \mathrm{O}_{3}$ as showed in Figs. 4 (b) and 4 (c), as a function of the time bins of the TDC with the time gate widths of 200 ps, 400 ps, 600 ps and 1200 ps, respectively. The purpose of this figure was to show that the higher resolution is needed to distinguish the spectra of two layers independently when the depth difference between the layers is shrunk. As can be seen in Fig. 6 (a), the maximum intensities of Raman spectra of sunflower oil and $\mathrm{Ba}\left(\mathrm{Mg}_{1 / 3} \mathrm{Ta}_{2 / 3}\right) \mathrm{O}_{3}$ can be observed within the second and fourth time bins, respectively corresponding to depth difference of $46 \mathrm{~mm}$. The actual depth difference between the layers was measured to be here $65 \mathrm{~mm}$. The Raman spectra of these two layers merge together already with the time gate width of 600 ps as shown in Fig. 6 (c). As already mentioned above, the IRF of the whole system is approximately 570 ps meaning that Raman scattered photons can be detected during this whole time period of 570 ps. This sets the required minimum depth difference between the two layers if their Raman spectra are wanted to be identified independently and explains why the spectra merge together with the wider time gate widths.

The depth derivation errors made with the samples containing $\mathrm{TiO}_{2}$ and $\mathrm{Ba}\left(\mathrm{Mg}_{1 / 3} \mathrm{Ta}_{2 / 3}\right) \mathrm{O}_{3}$ were $18.5 \mathrm{~mm}$ and 19 $\mathrm{mm}$, respectively, which are larger than the maximum

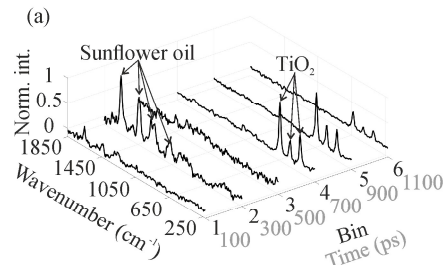

(c)

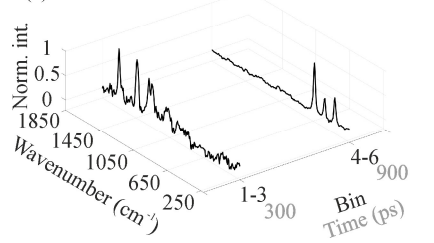

(b)

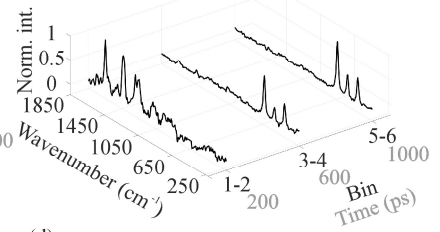

(d)

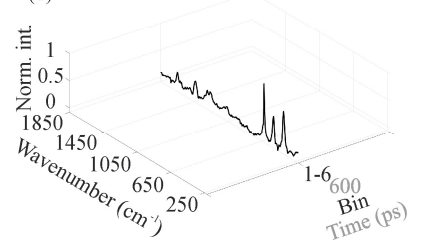

Fig. 5. The measured Raman spectra of the heterogenous liquid sample consisting of water between sunflower oil and $\mathrm{TiO}_{2}$ as a function of the time bins of the TDC with the time gate widths of (a) 200 ps, (b) 400 ps, (c) 600 ps and (d) 1200 ps.

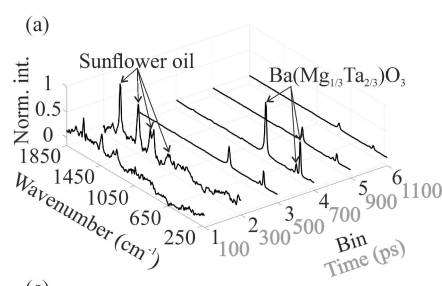$$
\text { (c) }
$$$$
\text { (b) }
$$
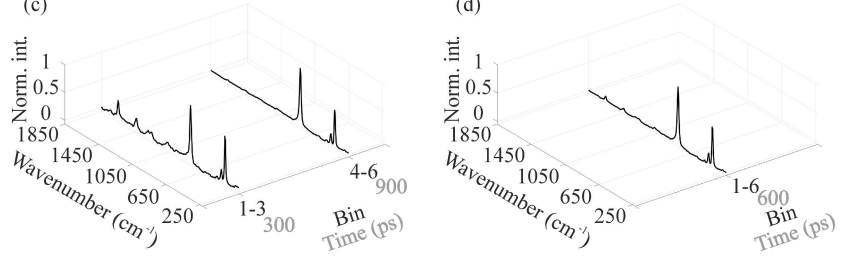

Fig. 6. The measured Raman spectra of the heterogenous liquid sample consisting of water between sunflower oil and $\mathrm{Ba}\left(\mathrm{Mg}_{1 / 3} \mathrm{Ta}_{2 / 3}\right) \mathrm{O}_{3}$ as a function of the time bins of the TDC with the time gate widths of (a) $200 \mathrm{ps}$, (b) 400 ps, (c) 600 ps and (d) 1200 ps.

quantization error $( \pm 200 \mathrm{ps} / 2 \Rightarrow>12 \mathrm{~mm})$. However, this larger error can be explained by the differential nonlinearities of the TDC and the timing skew of the time bins along the spectral axis. The timing skew of the sensor circuit has been measured to be \pm 75 ps over the whole spectral range [16]. This might cause that the measured ToA of Raman photons at different spectral points can have the maximum time error of 150 ps. Basically this could mean that if we have a layer that has Raman peaks at the both ends of the spectral axis, the maximum Raman intensity at the beginning of the spectral axis might be observed in a different time bin than the maximum intensity at the end of the spectral axis. Now in our measurement, $\mathrm{Ba}\left(\mathrm{Mg}_{1 / 3} \mathrm{Ta}_{2 / 3}\right) \mathrm{O}_{3}$ and $\mathrm{TiO}_{2}$ have their Raman peaks at the first half of the wavenumber axis whereas sunflower oil have its Raman peaks at the latter half and thus, the timing skew is causing some error to the accuracy of the depth derivation.

Fig. 7 shows the effective suppression of the fluorescence tail from the sunflower oil layer with different time gate widths in the case of first sample configuration (sunflower oilwater- $\mathrm{TiO}_{2}$ ). The maximum intensities of Raman peaks of $\mathrm{TiO}_{2}$ were observed within the fifth time bin as shown in Fig. 5 (a) and explained above. With the same time bin, the lowest fluorescence background level can be also observed because the most effective fluorescence tail suppression can be achieved with the shortest time gate width as shown in Fig. 7. The fluorescence background level increases when the Raman 


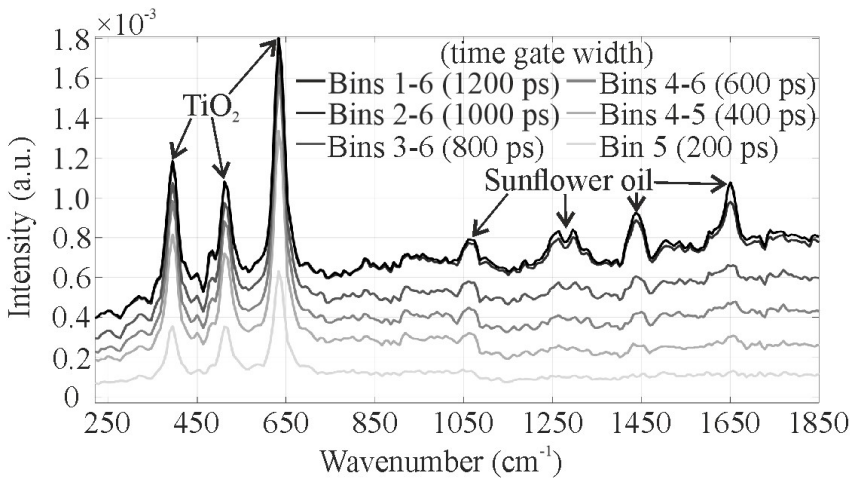

Fig. 7. Raman spectra of the the heterogenous liquid sample consisting of water between sunflower oil and $\mathrm{TiO}_{2}$ measured with the time gate widths from 200 ps to $1200 \mathrm{ps}$.

spectra are derived by using several time bins meaning that the maximum fluorescence level is observed when the full range of the TDC is used (Bins 1-6) as shown in Fig. 7. The fluorescence background levels are approximately equal when the time bins 1-6 and 2-6 are used because the first time bin is basically collecting just the rising edge of the Raman photons from sunflower oil. In addition, the increase of Raman photons from $\mathrm{TiO}_{2}$ can be observed when the time gate width is widened from $200 \mathrm{ps}$ to $600 \mathrm{ps}$ because the full width of the IRF of the whole system was 570 ps as mentioned above. As a result, to achieve the best depth resolving capability, the shortest time gate should be used, but the best possible signalto-noise ratio is achieved with the wider time gates. In some cases, it might be necessary to use a wider time gate to be even able to observe the Raman spectrum of a low Raman sensitive sample.

\section{CONCLUSIONS}

The single burst depth-resolving Raman spectrometer based on the $16 \times 256$ SPAD array with the on-chip 256channel TDC was presented in this paper. The depth derivation of the different layers of the heterogeneous liquid samples was based on measuring the ToA of backscattered Raman photons generated by a single burst of laser pulses by utilizing the on-chip TDC integrated in the same die with the SPAD line sensor. Raman spectra from different depths was derived by post-processing the time domain histograms in which the specific time bins corresponds to Raman photons from different depths. Measurements showed that the Raman spectra of two layers inside the sample with depth difference of $65 \mathrm{~mm}$ could be independently identified with the resolution of the TDC of $200 \mathrm{ps}$ ( $23 \mathrm{~mm}$ depth in water). To be able to identify independently the Raman spectra of two layers that are closer to each other, the impulse response function of the whole system should be improved from $570 \mathrm{ps}$ $(68 \mathrm{~mm})$ or otherwise the Raman spectra of the layers will merge together. The depth derivation error was measured to be less than $19 \mathrm{~mm}$, which is still larger than the maximum quantization error of the TDCs, but this could be explained by the timing skew and the nonlinearities of the TDCs along the spectral axis. The measurement also showed that the fluorescence tail of the top sample can be effectively suppressed by using the time gate width of $200 \mathrm{ps}$. On the other hand, longer 600 ps time gate width is need if all the Raman photons from a target layer are wanted to be collected due to the 570 ps full-width IRF of the device. The presented technology makes it possible to derive rapidly the Raman depth profiles of liquids and other semitransparent media with actual depth information by using only a single burst of laser pulses. This could open new application possibilities, for example, in quality control in the food and beverage industry and in the studying of natural and mining waters.

\section{REFERENCES}

[1] E.B. Hanlon, et. al. "Prospects for in vivo Raman spectroscopy, Topical Review", Phys. Med. Biol., vol. 45, pp. R1-R59, Feb. 2000.

[2] Nancy L. Jestel, "Process Raman Spectroscopy", in "Process Analytical Technology Spectroscopic Tools and Implementation Strategies for the Chemical and Pharmaceutical Industries", (Blackwell Publishing, 2005), Chap. 5.

[3] O. Khalil, "Spectroscopic and Clinical Aspects of Noninvasive Glucose Measurements", Clinical Chemistry, vol. 45, no. 2, pp. 165177, Feb. 1999.

[4] R.P. Van Duyne, D.L. Jeanmaire, and D.F. Shriver, "Mode-Locked Laser Raman Spectroscopy-A New Technique for the Rejection of Interfering Background Luminescence Signals," Analytical Chemistry, vol. 46, no. 2, pp. 213-222, Feb. 1974.

[5] P. Matousek et al., "Fluorescence suppression in resonance Raman spectroscopy using a high-performance picosecond Kerr gate," Journal of Raman Spectroscopy,vol. 32, no. 12,pp. 983-988, Dec. 2001.

[6] D.V. Martyshkin, R.C. Ahuja, A. Kudriavtsev, and S.B. Mirov, "Effective suppression of fluorescence light in Raman measurements using ultrafast time gated charge coupled device camera,"Review of Scientific Instruments, vol. 75, no. 3, pp. 630-635, Mar. 2004.

[7] P. Matousek, N. Everall, M. Towrie, and A. W. Parker, "Depth profilingin diffusely scattering media using Raman spectroscopy and picosecond kerr gating," Applied Spectroscopy, vol. 59, no. 2, pp. 200 205, Feb. 2005.

[8] J. Kekkonen, J. Nissinen and I. Nissinen, "Depth Analysis of SemiTransparent Media by a Time-Correlated CMOS SPAD Line SensorBased Depth-Resolving Raman Spectrometer," IEEE Sensors Journal, vol. 19 , no. 16, pp. 6711-6720, Aug. 2019.

[9] F. Ariese, H. Meuzelaar, M. M. Kerssens, J. B. Buijs, and C. Gooijer, "Picosecond Raman spectroscopy with a fast intensified CCD camera for depth analysis of diffusely scattering media," Analyst, vol. 134, no. 6, pp. 1192-1197, Mar. 2009.

[10] S. K. V. Sekar et al. , "Time domain diffuse Raman spectrometer based on a TCSPC camera for the depth analysis of diffusive media," Opt. Lett., vol. 43, no. 9, pp. 2134-2137, 2018.

[11] C. Zhang, L. Zhang, R. Yang, K. Liang, and D. Han, “Time-correlated Raman and fluorescence spectroscopy based on a silicon photomultiplier and time-correlated single photon counting technique," Appl. Spectrosc., vol. 67, no. 2, pp. 136-140, Feb. 2013.

[12] I. Nissinen et al., "A sub-ns time-gated CMOS single photon avalanche diode detector for Raman spectroscopy,"in Proc. IEEE ESSDERC'11, Finland, Helsinki, 2011, pp. 375-378.

[13] J. Kostamovaara et al., "Fluorescence suppression in Raman spectroscopy using a time-gated CMOS SPAD," Optics Express, vol. 21, no. 25, pp. 31632-31645, Dec. 2013.

[14] Y. Maruyama, J. Blacksberg, and E. Charbon, "A 1024x8, 700-ps Time-Gated SPAD Line Sensor for Planetary Surface Exploration With Laser Raman Spectroscopy and LIBS," IEEE Journal of Solid-State Circuits, vol. 49, no. 1, pp. 179-189, Jan. 2014.

[15] A. Kufcsak et al., "Time-resolved spectroscopy at 19,000 lines per second using a CMOS SPAD line array enables advanced biophotonics applications," Optics Express, vol. 25, no. 10, pp. 11103-11123, May 2017.

[16] I. Nissinen et al., "A 16×256 SPAD Line Detector With a 50-ps, 3-bit, 256-Channel Time-to-Digital Converter for Raman Spectroscopy," IEEE Sensors Journal, vol. 18, no. 9, pp. 3789-3798, May 2018.

[17] A. Philippidis, E. Poulakis, A. Papadaki and M. Velegrakis, "Comparative Study using Raman and Visible Spectroscopy of Cretan Extra Virgin Olive Oil Adulteration with Sunflower Oil," Analytical Letters, vol. 50, no. 7, pp. 1182-1195, Jun. 2017

[18] T. Ohsaka, F. Izumi, and Y. Fujiki, "Raman spectrum of anatase, TiO2," Journal of Raman Spectroscopy, vol. 7, no. 6, pp. 321-324, Dec. 1978.

[19] M.-Y. Chen et al., "Microwave properties of $\mathrm{Ba}\left(\mathrm{Mg}_{1 / 3} \mathrm{Ta}_{2 / 3}\right) \mathrm{O}_{3}$, $\mathrm{Ba}\left(\mathrm{Mg}_{1 / 3} \mathrm{Nb}_{2 / 3}\right) \mathrm{O}_{3}$ and $\mathrm{Ba}\left(\mathrm{Co}_{1 / 3} \mathrm{Nb}_{2 / 3}\right) \mathrm{O}_{3}$ ceramics revealed by Raman scattering," Journal of the European Ceramic Society, vol. 26, no. 1011, pp. 1965-1968, 2006. 